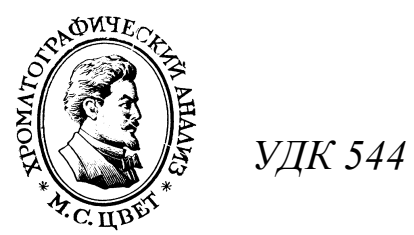

\title{
Приближение слабого градиента в теории градиентной ионной хроматографии
}

\author{
(c) 2020 Долгоносов А.М., Прудковский А.Г. \\ Институт геохимии и аналитической химии им. В.И. Вернадского Российской академии наук, Москва \\ Поступила в редакцию 31.10 .2020 г.
}

DOI: $10.17308 /$ sorpchrom.2020.20/3049

В работе приводятся основные соотношения для кинетических и равновесных характеристик градиентной ионной хроматографии. Найдена связь этих характеристик с параметрами компонентов на динамической карте хроматографической системы. Получены уравнения траектории, отображающей на карте ход хроматографического эксперимента. Сформулированы принципы и рассмотрены простейшие алгоритмы оптимизации градиентной ионной хроматографии. Показано, что, несмотря на чрезвычайную сложность, теоретическое описание градиентных режимов ионной хроматографии возможно с приемлемой точностью. Развитый подход к описанию сложных режимов ионной хроматографии позволяет выявить новые тонко настраиваемые условия (например, знакопеременные градиентные режимы типа «линзы»), повышающие производительность хроматографии. Достоинством предложенного в работе режима «линза» является равенство состава элюента в начале и в конце процесса, что позволяет исключить регенерацию колонки для повторного анализа. К трудностям таких режимов можно отнести необходимость очень точного расчета и выполнения функции смешения, задающей зависимость силы элюента от времени. Решению подобных проблем служит имитационное моделирование, в результате которого создана компьютерная программа IONCHROM, позволяющая спрогнозировать поведение системы в найденных условиях. Разобран пример реальной системы, для которой предсказан режим «линза», являющийся специальным образом организованным знакопеременным градиентным режимом.

Ключевые слова: теория ионной хроматографии, градиентный режим, оптимизация разделения.

\section{Введение}

Выбор наилучших условий разделения смесей с учетом селективности и эффективности хроматографической системы - центральная проблема оптимизации хроматографии. В литературе выработан ряд критериев оптимальности, направленных на достижение максимального разделения за определенное время анализа, максимальной производительности анализа и т.п. $[1,2]$. Важным критерием оптимизации является понятие пиковой емкости, введенной в работах Кайзера [3] как число (оно может быть нецелым) дополнительных пиков, которые можно поместить в свободных от пиков промежутках хроматограммы. Поиск минимума пиковой емкости соответствует цели повышения производительности хроматографии. Однако пиковая емкость - не очень удобный критерий оптимизации, так как строго нулевая пиковая емкость практически недостижима, а результат с ненулевой пиковой емкостью является вырожденным и нуждается в дополнительных критериях. Более точным критерием является время однократного анализа при выполнении требований по степени разделения компонентов $[4,5]$. Эффективное применение этого критерия требует наличия математической модели хроматографического метода. 
В работах [5-7] рассмотрены принципы и возможности подхода к моделированию ионной хроматографии (ИХ), базирующегося на достижениях теории динамики сорбции, адаптированной к особенностям ионной хроматографии. В результате компьютерного моделирования была разработана программа IonChrom ${ }^{\mathbb{0}}$ [8]. C ee помощью были рассмотрены задачи многоколоночной ИХ с изократическим элюированием [9].

Градиентное элюирование является удобным способом анализа смесей с компонентами, сильно различающимися по удерживанию [10]. В немногочисленных работах по моделированию градиентной хроматографии $[11,12]$ разрабатываются простые модели удерживания, позволяющие рассчитывать однокомпонентные элюенты и линейные градиенты. Однако уровня этих моделей явно недостаточно для рассмотрения сложных градиентных режимов, слабо учитывается достигаемая в расчетах степень разделения компонентов пробы, что сильно обесценивает роль моделирования при разработке методик и при анализе (соответственно при решении прямой и обратной задач). В работах $[13,14]$ приводятся основные соотношения для равновесных и кинетических характеристик градиентной ИХ, даны примеры решения прямой и обратной задач для систем ионных хроматографов фирмы DIONEX, использующих как изократическое, так и градиентное элюирование. Дальнейшие исследования $[15,16]$ показали, что точность описания градиентной хроматографии можно повысить. Уровень детализации модели градиентной хроматографии в программе IONCHROM достаточно высокий для рассмотрения знакопеременных градиентных режимов [17-19].

Предлагаемая работа указывает на возможность создания универсального алгоритма решения чрезвычайно сложной задачи оптимизации градиентного режима ионной хроматографии на примере так называемого режима «слабых» градиентов. Для наглядности все выкладки проведены для «простых» компонентов, имеющих в изучаемых условиях единственную ионную форму. Переход к полиморфным компонентам не представляет принципиальных трудностей (см. [5-7]).

\section{Краткие сведения о математической модели ИХ}

Уравнения динамики сорбции в линейном приближении, характерном для процесса ИХ в разделяющей колонке, имеют вид $[5,20]$ :

$$
\begin{gathered}
\theta_{i} \frac{\partial a_{i}}{\partial t}=\Gamma_{i} c_{i}-a_{i} \\
\varepsilon \frac{\partial c_{i}}{\partial t}+v \frac{\partial c_{i}}{\partial x}+\frac{\partial a_{i}}{\partial t}=\frac{\partial}{\partial x}\left(D_{l i} \frac{\partial c_{i}}{\partial x}\right)
\end{gathered}
$$

где $t$ и $x$ - время и координата вдоль колонки; $c_{i}$ - мольная концентрация компонента пробы на единицу объема подвижной фазы (элюента); $a_{i}$ - мольная концентрация сорбированного вещества пробы на единицу объема колонки; $\theta_{i}$ - характерное время сорбции, определяемое коэффициентами внешней и внутренней диффузии, размерами зерен сорбента и их внутренним строением; $\varepsilon$ - порозность (доля в колонке объема элюента (подвижной фазы, ПФ)); $v=\frac{4 w}{\pi d^{2}}-$ линейная скорость ПФ, рассчитанная на полное сечение колонки диаметром $d$, при скорости потока $w ; D_{l i}-$ коэффициент продольной диффузии; $\Gamma_{i}-$ коэффициент распределения $i$-го компонента 
пробы. Для простого компонента пробы (т.е. компонента с единственной ионной формой)

$$
\Gamma_{i}=\frac{K_{i}^{z_{i}}}{\varphi^{z_{i}}},
$$

где $K_{i} \equiv K_{R, i}$ - константа ионного обмена $i$-го компонента пробы на некоторый однозарядный ион сравнения, или опорный ион (обозначен как $R$ ), $z_{i}$ - заряд ионов пробы, $\varphi$ - сила элюента, которая определяется его качественным и количественным составом по следующему уравнению:

$$
a_{0}=\sum_{j, s} s a_{j_{s}}=\sum_{j, s} s f^{s} c_{j_{s}} K_{j_{s}}^{s} \varphi^{-s},
$$

где $a_{0}=$ const - емкость неподвижной фазы (сорбента), $a_{j_{s}}, c_{j_{s}}-$ концентрации ионной формы с зарядом $s j$-го компонента элюента в неподвижной и подвижной фазах, соответственно; $K_{j_{s}} \equiv K_{R, j_{s}}$ - соответствующие константы ионного обмена; $f$ - результирующая величина коэффициента активности, не сильно отличающаяся от 1 и определяемая по Дебаю-Хюккелю [21]. В градиентных режимах сила элюента, так же как и концентрации компонентов элюента $c_{j}$, зависит от времени, $\varphi(t)$.

Решение системы (1) возможно с помощью метода моментов [15, 16]. Выражение для первого момента связывает путь, пройденный зоной аналита в колонке, с временем:

$$
l_{i}(t)=v \int_{0}^{t} \frac{d \xi}{\Gamma_{i}(\xi)}
$$

Из связи (4) можно определить обратную функцию $t_{i}(l)$. При $l=L$ ( $L-$ длина разделяющей колонки) функция $t_{i}(L)$ численно совпадает с исправленным временем удерживания $i$-го компонента $t_{i}^{\prime}$, равным разности $t_{i}^{\prime}=t_{i}-t_{0}$ времени удерживания пика $\left(t_{i}\right)$ и времени выхода свободного объема хроматографической системы (мертвого времени $t_{0}$ ). Формула для второго момента решения,

$$
\sigma_{i}^{2}=\frac{\Gamma_{i}^{2}\left(t_{i}^{\prime}\right)}{v} \int_{0}^{t_{i}^{\prime}} h_{i}(t) \frac{\left[\Gamma_{i}(t)+\varepsilon\right]^{2}}{\Gamma_{i}^{3}(t)} d t,
$$

определяет временную дисперсию $i$-го пика пробы на выходе из разделяющей колонки. Высота, эквивалентная теоретической тарелке (ВЭТТ), является локальной величиной:

$$
h_{i}(t)=\frac{2 v \Gamma_{i}(t) \theta_{i}(t)}{\left[\Gamma_{i}(t)+\varepsilon\right]^{2}}+\frac{2 D_{l i}(t)}{v} .
$$

ВЭТТ удобна в вычислениях тем, что она сравнительно медленно изменяется при вариациях коэффициента распределения $\Gamma_{i}$.

При изократическом хроматографическом режиме состав элюента постоянен во времени и тогда формулы (4), (5) упрощаются:

$$
\begin{gathered}
L=\frac{v}{\Gamma_{i}} t_{i}^{\prime} \\
\sigma_{i}^{2}=h_{i} \frac{\left(\Gamma_{i}+\varepsilon\right)^{2}}{v \Gamma_{i}} t_{i}^{\prime}=L h_{i} \frac{\left(\Gamma_{i}+\varepsilon\right)^{2}}{v^{2}}=\frac{t_{i}^{2}}{N_{i}}
\end{gathered}
$$


где $N_{i}=L / h_{i}$ - эффективность (число теоретических тарелок) по данному компоненту, так же, как и ВЭТТ, слабо зависящая от $\Gamma_{i}$. С учетом этого из формулы (8) следует, что условия, контролирующие изменение $t_{i}$, приводят в первом приближении к пропорциональному изменению $\sigma_{i}$. Иными словами, градиентный хроматографический режим с изменяемым элюентом сопровождается масштабным преобразованием хроматограммы (тем большим сжатием, чем сильнее элюент).

\section{Динамическая карта хроматографической системы}

Систему уравнений (2)-(5) можно представить в виде графика - динамической карты хроматографической системы (ДКХС). ДКХС удобна как характеристика и инструмент исследования хроматографического поведения интересующих компонентов. На рис.1 приведена ДКХС для смеси анионов в модельной системе с характеристиками хроматографа фирмы DIONEX [10].

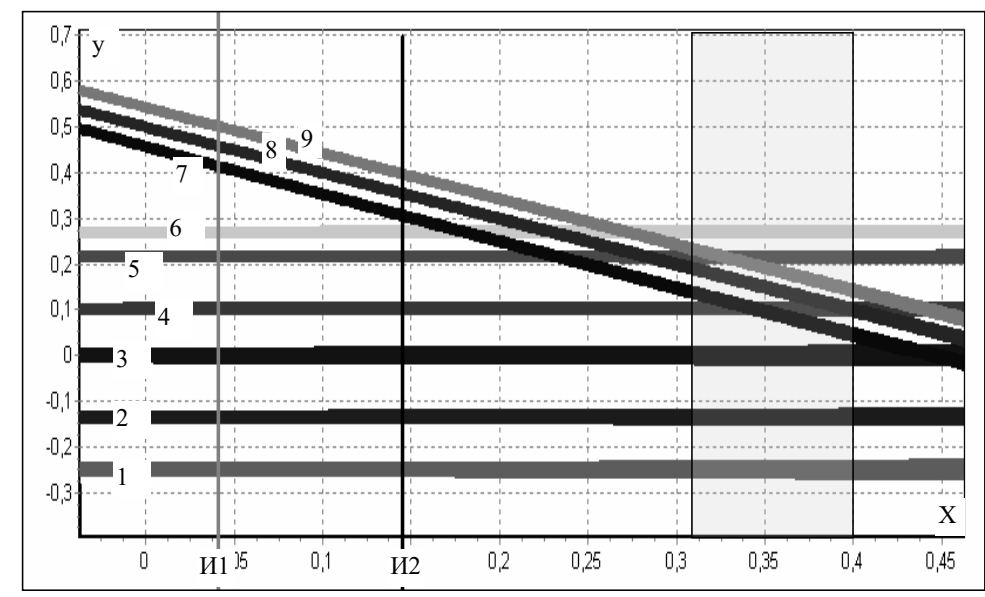

Рис. 1. Динамическая карта хроматографической системы для разделения с критерием $\mathrm{R}=1$ смеси анионов: (1) $\mathrm{F}^{-},(2) \mathrm{HCOO}^{-},(3) \mathrm{Cl}^{-},(4) \mathrm{NO}_{2}^{-},(5) \mathrm{Br}^{-},(6) \mathrm{NO}_{3}^{-}$, (7) $\mathrm{PO}_{4}{ }^{3-},(8) \mathrm{PO}_{3} \mathrm{~F}^{2-},(9) \mathrm{SO}_{4}{ }^{2-},-$ при $\mathrm{pH} 10.82$ и следующих характеристиках: разделяющая колонка 180x3 мм, сорбент со структурой поверхностно-привитого ионита AS-14a зернением $5 \pm 0.5$ мкм, с толщиной сферической оболочки 140 нм, емкостью 0.038 мэкв/ $\mathrm{cm}^{3}$; подавитель-электродиализатор ASRS-ULTRA2-mm; расход карбонатного элюента $0.5 \mathrm{~cm}^{3} /$ мин [10]. Вертикальные линии соответствуют двум изократическим режимам: И1 - приведенному в каталоге фирмы DIONEX эксперименту, И2 - оптимальному элюенту в заданных условиях. Выделенный штриховкой прямоугольник (в запрещенной области для изократики с критерием $\mathrm{R}=1$ ) соответствует границам слабого градиентного режима, показанного на рис.3.

Fig. 1. Dynamic map of the chromatographic system (DMCS) for separation with the criterion $\mathrm{R}=1$ of anion mixture: (1) $\mathrm{F}^{-}$, (2) $\mathrm{HCOO}^{-}$, (3) $\mathrm{Cl}^{-}$, (4) $\mathrm{NO}_{2}^{-}$, (5) $\mathrm{Br}^{-}$, (6) $\mathrm{NO}_{3}^{-}$, (7) $\mathrm{PO}_{4}{ }^{3-}$, (8) $\mathrm{PO}_{3} \mathrm{~F}^{2-}$, (9) $\mathrm{SO}_{4}{ }^{2-}$, - at $\mathrm{pH} 10.82$ and the following characteristics: separating column 180x3 mm, sorbent with the structure of surface-grafted ion exchanger AS-14a with grain size of $5 \pm 0.5 \mu \mathrm{m}$, with a spherical shell thickness of $140 \mathrm{~nm}$, and a capacity of $0.038 \mathrm{mEq} / \mathrm{ml}$; suppressor electrodialysis cell ASRS-ULTRA2-mm; the consumption of the carbonate eluent is $0.5 \mathrm{ml} / \mathrm{min}$ [10]. The vertical lines correspond to two isocratic modes: I1 - the experimental mode, provided in the DIONEX catalogue, I2 - the optimal eluent under the specified conditions. The shaded rectangle (in the forbidden region for isocracy with the criterion $\mathrm{R}=1$ ) corresponds to the boundaries of the weak gradient mode shown in Fig. 3. 
Абсциссой на динамической карте является $X=\lg \varphi$, где сила элюента $\varphi$ вычисляется по уравнению (3). На оси ординат (ось $y$ ) откладывается логарифм относительного удерживания, если за нуль принята характеристика опорного компонента:

$$
y_{i}=\lg \left(\Gamma_{i} / \Gamma_{R}\right)
$$

В общем случае многокомпонентного элюента водородный показатель $p H$ может рассматриваться как независимая переменная и ДКХС представляет собой сечение зависимости $y(X, p H)$ плоскостью $p H=$ const . Каждому компоненту пробы на ДКХС соответствует полоса, построенная вокруг средней линии $y_{i}(X)$, которая согласно (2) описывается уравнением:

$$
y_{i}=z_{i} \lg K_{i}-\left(z_{i}-1\right) X,
$$

где учтены единичные значения константы и заряда для опорного иона.

Границы полосы связаны с характеристиками хроматограммы - исправленным временем удерживания $t_{i}^{\prime}$ и полушириной пика $\tau_{i}=\sigma_{i} \sqrt{8 \ln 2} \approx 2.35 \sigma_{i}$ - выражением

$$
y_{i \pm}=y_{i}+\lg \left(1 \pm \delta_{i}\right) \approx y_{i} \pm \delta_{i} \lg e, \delta_{i}=R_{i} \tau_{i} / t_{i}^{\prime} \approx R_{i} \sqrt{8 \ln 2 / N_{i}}<<1,
$$

где приближенные равенства относятся к участкам полос с параллельными границами (в левой части ДКХС), $\delta_{i} \lg e \approx 1.02 R_{i} / \sqrt{N_{i}}$ - полуширина $i$-ой полосы, $R_{i}$ - требуемая степень (критерий) разделения пиков этого и соседних компонентов. Зоны пересечения полос на ДКХС соответствуют пикам на хроматограмме, не разделенным с необходимой степенью.

Отметим, что на ДКХС можно отразить только исправленное (или смещенное) время, отличающееся от значений на хроматограмме на постоянную величину мертвого времени $t_{0}$. Такой же аргумент имеет функция смешения, задающая зависимость силы элюента от времени - последняя участвует в уравнениях в виде функции $\varphi\left(t^{\prime}\right)$.

Из уравнения (10) следует, что ионы с различающимися зарядами всегда можно разделить, подобрав соответствующий режим на динамической карте, так как их полосы идут под разными углами и, начиная с некоторой точки, перестают пересекаться. Также можно заметить, что с разных сторон от точки пересечения средних линий лежат области режимов с разным порядком элюирования этих компонентов.

Средние линии двух одинаково заряженных ионов пробы параллельны, так что их последовательность выхода не может быть изменена. От величины силы элюента зависит только ширина полос. Рассмотрим в этом случае условие пересечения полос.

Степень разделения $R_{i j}$ компонентов с сопоставимыми концентрациями выражается формулой:

$$
R_{i j}=\frac{\left|t_{i}^{\prime}-t_{j}^{\prime}\right|}{\tau_{i}+\tau_{j}}
$$

Для осуществления требуемого разделения необходимо, чтобы минимальная степень разделения $i$-го компонента со своими соседями была бы не меньше его критерия разделения:

$$
R_{i} \leq \min _{j} R_{i j}
$$

Передвижение зоны компонента пробы по колонке подчиняется уравнению (4). Для простого компонента подстановка (2) в (4) дает интегральное уравнение: 


$$
\int_{0}^{t_{i}^{\prime}} \varphi^{z_{i}}(t) d t=\frac{L}{v} K_{i}^{z_{i}}
$$

Результатом решения уравнения (14) является зависимость $t^{\prime}(K, z)$. Учитывая, что у соседних равнозарядных компонентов константы обмена близки друг к другу, из соотношения (14) получим:

$$
t_{i+1}-t_{i} \approx \frac{L}{v \varphi^{z}\left(t_{i}^{\prime}\right)}\left(K_{i+1}^{z}-K_{i}^{z}\right)
$$

Для изократического режима из формул (8), (12), (15), учитывая, что дисперсии обоих пиков приближенно одинаковы, следует:

$$
R_{i, i+1} \approx 0.212 \sqrt{N_{i}} \frac{K_{i+1}^{z}-K_{i}^{z}}{K_{i}^{z}+\varepsilon \varphi^{z}}
$$

Заметим, что согласно (16) при росте $X=\lg \varphi$ степень разделения одинаково заряженных компонентов уменьшается. На ДКХС этот вывод отражается в уширении параллельных полос при движении вправо, т.е. всегда можно найти такую область значений силы элюента, в которой они пересекаются.

Итак, если на ДКХС имеются области выполнения условия требуемого разделения заданной пары компонентов (13), то существует, по меньшей мере, одна точка, где реализуется равенство степени разделения и нижней границы критерия разделения для каждого из них. Эти точки являются ориентирами для построения траектории хроматографического опыта.

\section{Траектория хроматографического опыта на динамической карте}

Траектория какого-либо хроматографического опыта на ДКХС - это линия, пересечение которой с полосами компонентов соответствует их пикам на хроматограмме этого опыта. В частности, значения времени для точек пересечения траектории со средними линиями полос равны исправленным временам удерживания соответствующих компонентов. Для изократических режимов на динамической карте в силу (2), (7) можно определить функции:

$$
y\left(X, t^{\prime}\right)=X+\lg t^{\prime}-\lg (L / v)
$$

Так как $\lg (L / v)=$ const, то отсюда следует, что изохрона - линия $t^{\prime}=$ const, направлена под углом $45^{\circ}$ к оси $X$, т.е. время растет при движении по карте вверх и влево.

Уравнение (17) задает вид траектории изократического режима. Характерной чертой изократического режима является постоянство силы элюента $X=X_{I}=$ const , поэтому искомая траектория представляет собой «вертикальную» линию - прямую, параллельную оси $y$, проведенную через точку $X_{I}$ (рис.1). Для сечения карты этой линией получим хроматограмму, время которой отсчитывается от начала опыта и поэтому смещено относительно времени на ДКХС на величину мертвого времени. На рис.2а сравниваются результаты такого расчета и соответствующего эксперимента, приведенного фирмой DIONEX. На рис. 26 дана теоретическая хроматограмма, рассчитанная для режима И2, соответствующая оптимальному изократическому разделению на описанной в подписи к рис.1 экспериментальной системе.

Для градиентного режима, решая совместно уравнения (10) для средней линии полосы и (14) для времени удерживания, получим функцию: 


$$
y\left(X, t^{\prime}\right)=X+\lg r_{z}\left(t^{\prime}\right)-\lg \frac{L}{v}
$$

где $r_{z}\left(t^{\prime}\right)=\varphi^{-z}\left(t^{\prime}\right) \int_{0}^{t^{\prime}} \varphi^{z}(\xi) d \xi$. Зависимость $X\left(t^{\prime}\right) \equiv \lg \varphi\left(t^{\prime}\right)$ для абсциссы траектории градиентного режима известна (задана уравнением (3)), а уравнение для ординаты траектории получим, подставив эту зависимость в (18). Следствиями (18) являются: выражение (17) - при $\varphi=$ const, а также параллельность изохрон карты $t^{\prime}=$ const для градиентного и изократического режимов. Для градиентного элюирования обычно характерна возрастающая функция $\varphi\left(t^{\prime}\right)$, приводящая к неравенству $r_{z}\left(t^{\prime}\right)<t^{\prime}$. Таким образом, точка на карте с координатами $X, y$ при положительном градиенте соответствует большему времени, чем при изократике, и наоборот - вывод, важный при оптимизации.
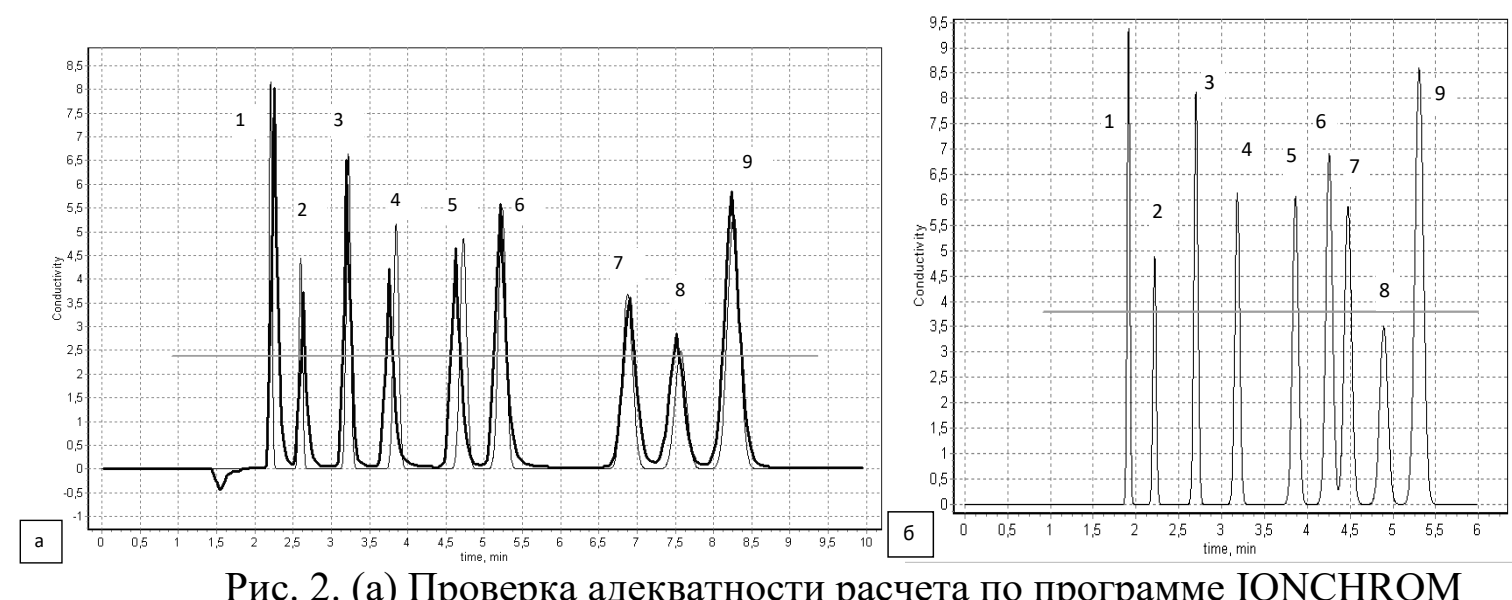

Рис. 2. (a) Проверка адекватности расчета по программе IONCHROM при сравнении теоретической хроматограммы для режима И1 (тонкая линия) с экспериментальной хроматограммой (жирная линия, [10]). (б) Теоретическая хроматограмма (расчет для режима И2), соответствующая оптимальному изократическому разделению. Обозначения и параметры хроматографической системы указаны в подписи к рис.1. Горизонтальная линия на поле хроматограммы показывает характер функции смешения элюента $\varphi(t)$.

Fig. 2. (a) Checking the adequacy of the calculation using the IONCHROM program when comparing the theoretical chromatogram for the I1 mode (thin line) with the experimental chromatogram (thick line, [10]). (b) Theoretical chromatogram (calculation for the I2 mode) corresponding to the optimal isocratic separation. The designations and parameters of the chromatographic system are indicated in the caption to Fig. 1. The horizontal line in the chromatogram field shows the nature of the eluent mixing function $\varphi(t)$.

Для линейной зависимости $X\left(t^{\prime}\right)=X_{0}+b t^{\prime}$ (т.е. при экспоненциальной зависимости концентрации элюента от времени) из (18) получим уравнение:

$$
y(X)=X+\lg \left[1-10^{-z\left(X-X_{0}\right)}\right]-\lg (b z \ln 10)-\lg \frac{L}{v}
$$

Интересны следствия (19) для больших и малых величин постоянной $b$. В первом случае имеет место приближенное равенство:

$$
y(X) \approx X-\lg (b z \ln 10)-\lg \frac{L}{v}, b t^{\prime}>>1 / z
$$


Согласно (20) траектории асимптотически стремятся к изохронам для малых времен, сдвинутым вниз друг относительно друга на логарифм отношения зарядов. В этом приближении все пики равнозарядных компонентов не разделяются, а могут быть разделены только компоненты с разными зарядами: порядок выхода будет от больших зарядов к меньшим. Константы обмена роли не играют, поэтому этот режим представляет ограниченный интерес.

Для малых величин $b$ получим приближенное выражение, не зависящее от зарядов:

$$
y(X) \approx X+\lg \frac{X-X_{0}}{b}-\lg \frac{L}{v}, \quad b t^{\prime}<<\lg e
$$

В этом случае, характерном для времен $\frac{X-X_{0}}{b}$, траектория едина для всех компонентов. Можно показать, что для малого полного смещения $X-X_{0}$ вид функции $X\left(t^{\prime}\right)$ (или обратной ей функции $\left.t^{\prime}(X)\right)$ роли не играет; в этом случае получим уравнение для траектории «слабого» градиентного режима, совпадающее по форме с уравнением изократики (17):

$$
y(X) \approx X+\lg t^{\prime}(X)-\lg \frac{L}{v}
$$

Уравнение (22) позволяет объединить подходы к описанию изократического и градиентного режимов путем использования неизменной динамической карты и единой для любых ионов траектории, отвечающей конкретной реализации хроматографии. В этом приближении различие в изохронах для разных режимов незначимо: можно считать, что характеристики точки на ДКХС не зависят от пути ее достижения. На практике, при построении траектории слабого градиентного режима следует выбирать участки с минимальным наклоном по отношению к оси ординат.

Итак, очевидны следующие свойства траекторий. Траектория изократического режима параллельна оси ординат. Наклон любого участка траектории градиентного режима относительно этой оси не может превышать наклона изохрон $\left(45^{\circ}\right)$, так как время эксперимента не может двигаться вспять. Более того, так как любой участок хроматографического процесса имеет ненулевую длительность, то изохроны не могут быть элементами траектории. Наклонные участки траектории ветвятся на линии, соответствующие разным зарядам, - тем заметнее, чем больше наклон и длина участка. В первом приближении этот эффект незначителен, что позволяет рассматривать траекторию слабого градиентного режима, как единую для всех компонентов.

В общем случае выражение (18) дает разные траектории для одного и того же градиентного режима в зависимости от зарядов компонентов пробы. Появление на ДКХС пучка траекторий, каждая линия которого предназначена для ионов определенного заряда, усложняет картину; точки пересечения полос разнозарядных ионов перестают строго соответствовать точкам минимума между их пиками на хроматограмме. Выход из этой ситуации существует: он состоит в переопределении ДКХС, пересчете и дроблении карты при каждом шаге градиентного режима. Такой сложный, но универсальный путь реализован в программе IONCHROM [18, 20]. Ниже приведены основные моменты разработанного алгоритма.

Определим траекторию $y\left(t_{i}^{\prime}\right)$ для произвольного компонента $i$ формулой:

$$
y_{i}\left(t^{\prime}\right)=\lg \left[\frac{v}{L} \int_{0}^{t_{i}^{\prime}} 10^{X(\xi)} d \xi\right],
$$

где исправленное время удерживания $i$-го компонента $t_{i}^{\prime}$ определяется по формуле 
(7), а ордината траектории $y\left(t_{i}^{\prime}\right)$ соответствует ординате некого тестового однозарядного компонента с такой константой обмена $K_{i}$, чтобы их исправленные времена удерживания совпадали: $y_{i}\left(t^{\prime}\right)=\lg K_{i}$. Таким образом, определение (23) обеспечивает главное свойство ДКХС: пересечение полос на карте соответствует реальному пересечению пиков на хроматограмме.

Ординаты полос многозарядных компонентов зависят не только от времени, но и от формы траектории, поэтому при построении траектории они могут изменяться. В связи с этим, при поиске нужного режима построение градиентной карты ведется по шагам в прямом взаимодействии с компьютерной моделью процесса. Алгоритм такого процесса может быть следующим [18].

1. Начинаем построение с изократической ДКХС

2. Строим произвольный малый отрезок будущей траектории с положительным приращением координаты $\Delta y>0$ и произвольным $\Delta X$ : $X_{M}=X+\Delta X, y_{M}=y+\Delta y$.

3. По формуле (23) рассчитываем очередную точку $t_{M}^{\prime}$ на траектории, задаваемой функцией $X\left(t^{\prime}\right) \equiv \lg \varphi\left(t^{\prime}\right)$ :

$$
X_{M}=X\left(t_{M}^{\prime}\right), y_{M}=\lg \left[\frac{v}{L} \int_{0}^{t_{M}^{\prime}} 10^{X(\xi)} d \xi\right] \approx \lg \left[\frac{v}{L} \frac{\left(10^{X}+10^{X+\Delta X}\right)}{2} t_{M}^{\prime}\right]
$$

4. Процесс построения градиентной карты для момента времени $t_{M}^{\prime}$ начинается с разделения карты условной горизонтальной линией $y_{M}$. Под этой линией полосы уже вышедших компонентов, изменить времена выхода и ширину полос которых невозможно, над этой линией траекторию ещё предстоит построить. Для каждого $X$ строим карту ещё не вышедших компонентов в соответствии с формулой (23), условно считая, что для каждого $X$ при $t^{\prime}>t_{M}^{\prime}$ режим будет постоянным:

$$
y_{i}\left(t^{\prime}\right)=\lg \left\{10^{y_{M}}+10^{X} \frac{v}{L}\left(t_{i}^{\prime}-t_{M}\right)\right\},
$$

где время $t_{i}^{\prime}$ находится из уравнения:

$$
L=v \int_{0}^{t_{i}^{\prime}} \frac{d \xi}{\Gamma_{i}(X(\xi))}=v \int_{0}^{t_{M}^{\prime}} \frac{d \xi}{\Gamma_{i}(X(\xi))}+\frac{v\left(t_{i}^{\prime}-t_{M}^{\prime}\right)}{\Gamma_{i}(X)},
$$

Подставляя $\left(t_{i}^{\prime}-t_{M}^{\prime}\right)=\Gamma_{i}(X)\left[\frac{L}{v}-\int_{0}^{t_{M}^{\prime}} \frac{d \xi}{\Gamma_{i}(X(\xi))}\right]$ в (25), получим для каждого фиксированного $X$ :

$$
y_{i}\left(t^{\prime}\right)=\lg \left\{10^{y_{M}}+10^{X} \Gamma_{i}(X)\left(1-\frac{v}{L} \int_{0}^{t_{M}^{\prime}} \frac{d \xi}{\Gamma_{i}(X(\xi))}\right)\right\}
$$

5. Формула (24) определяет центры полос компонентов на виртуальной карте при $t^{\prime}>t_{M}^{\prime}, y>y_{M}$. Для построения полос на карте осталось для каждого фиксированного значения абсциссы $X$ вычислить ширину полос по формулам (7), (11).

6. Для $t^{\prime}>t_{M}^{\prime}, y>y_{M}$ делаем очередной шаг, возвращаясь к п.2

\section{Проблема оптимизации состава подвижной фазы}

Задача оптимизации хроматографического анализа может быть определена как поиск параметров системы для осуществления наиболее быстрого разделения 
заданной смеси с заданными критериями разделения для каждого компонента. Таким образом, оптимум соответствует минимальному времени анализа при заданных ограничениях. В частности, к ограничениям может относиться и порядок элюирования компонентов. Внутри задачи оптимизации хроматографического анализа, при фиксированных параметрах хроматографа рассматривается задача выбора оптимального элюента. Основным параметром ПФ является сила элюента. Как говорилось выше, в случае многокомпонентных элюентов еще одним аргументом задачи оптимизации элюента является $p H$, поэтому общий оптимум определяется на плоскости $(p H, X)$.

У градиентного режима больше степеней свободы, чем у изократического, поэтому в задаче оптимизации кроме интегрального критерия, времени анализа, необходим локальный критерий, такой как пиковая емкость хроматограммы. Снижение пиковой емкости на каждом участке хроматограммы приводит в итоге к снижению времени анализа. Назовем идеальнылм градиентный режим, приводящий к нулевой пиковой емкости (имеется в виду отсутствие свободных участков на хроматограмме с полностью разделенными пиками). Такой режим соответствует абсолютному оптимуму при хроматографии заданной смеси в заданной системе - наименьшему времени анализа при требуемой степени разделения компонентов.

Свойства ДКХС и уравнения траектории (22) позволяют наглядно определять оптимальную силу ПФ в процессе изократического режима, просто сдвигая вертикальную линию, соответствующую выбранному порядку элюирования компонентов пробы, вправо, до пересечения полос на карте (линия И2 на рис.1). Для иллюстрации полезности этой процедуры на рис.2б приведена хроматограмма, рассчитанная для оптимального режима И2. В этих условиях время анализа сокращается в 1.5 раза.

Обратим внимание на важную деталь: при подходе слева к точке пересечения полос сохраняется такой порядок элюирования, при котором

$$
z_{i} \leq z_{i+1}
$$

где компоненты пронумерованы в порядке выхода из колонки. Итак, для оптимального изократического режима при условии, что значение критерия оптимальности (время анализа $t_{A}$ ) растет с ростом критерия разделения,

$$
\partial t_{A} / \partial R_{i}>0, \partial t_{A} / \partial R_{i+1}>0,
$$

всегда выполняется правило (28). Если компонент не подчиняется этому правилу, для него выполняется тождество:

$$
\partial t_{A} / \partial R_{j} \equiv 0
$$

Назовем компоненты, для которых верно условие (30), «некритичными», а остальные - «критичными» (по отношению к оптимизации). Критичные компоненты принадлежат к парам, для которых условие (13) имеет смысл равенства. Те из них, которые удовлетворяют условию (29), назовем главныли критичными компонентами. Условно критичные компоненты удовлетворяют одновременно равенству (13) и условию (30) в области отрицательных вариаций аргумента. При снижении требований к их разделению условно критичные компоненты переходят в разряд некритичных компонентов. Вся анализируемая проба может быть представлена как набор главных, условно критичных и некритичных компонентов. В идеальном оптимальном режиме с минимально возможным временем разделения смесь должна содержать только критичные компоненты.

Определим на ДКХС «разрешенные» интервалы как отрезки на оси абсцисс, на которых нет пересечений полос. Разрешенный интервал ограничен слева пересечением полос условно критичных, а справа - главных критичных компонентов. Соответственно, «запрещенные» отрезки дополняют разрешенные интервалы до пол- 
ной оси абсцисс. Разрешенные интервалы соответствуют разным последовательностям выхода компонентов. Максимально удовлетворяющим принципу идеальной оптимизации в изократических условиях является крайний правый разрешенный интервал на ДКХС с соответствующей последовательностью выхода компонентов.

Для градиентной хроматографии удобно ввести частично разрешенные интервалы $k$-го порядка с непересекающимися полосами первых $k$ компонентов. В целях минимизации локальной пиковой емкости построение траектории градиентного режима должно начинаться с отрезка траектории изократики, соответствующего правой точке такого интервала. Далее траектория проходит через точку пересечения полос более удерживаемых компонентов. Число таких отрезков на полученной траектории будет соответствовать числу пар критичных компонентов, плюс заключительный наклонный отрезок - от последней точки пересечения полос до точки окончания эксперимента. Параметры последнего в рамках теории слабого градиентного режима не определяются, однако они без труда рассчитываются численно.

Для поиска слабого градиентного режима, существенно улучшающего параметры хроматографии по сравнению с оптимальным изократическим режимом, рассмотрим вместо вертикальных прямых - траекторий изократического режима - вертикальные полосы небольшой ширины. Ширина полосы ограничена рамками принятого приближения. Такая полоса должна содержать искомую траекторию, что означает наличие внутри полосы всех точек пересечения полос критичных компонентов. Сама же траектория слабого градиентного режима имеет вид «змейки», направленной снизу-вверх (рис.3).

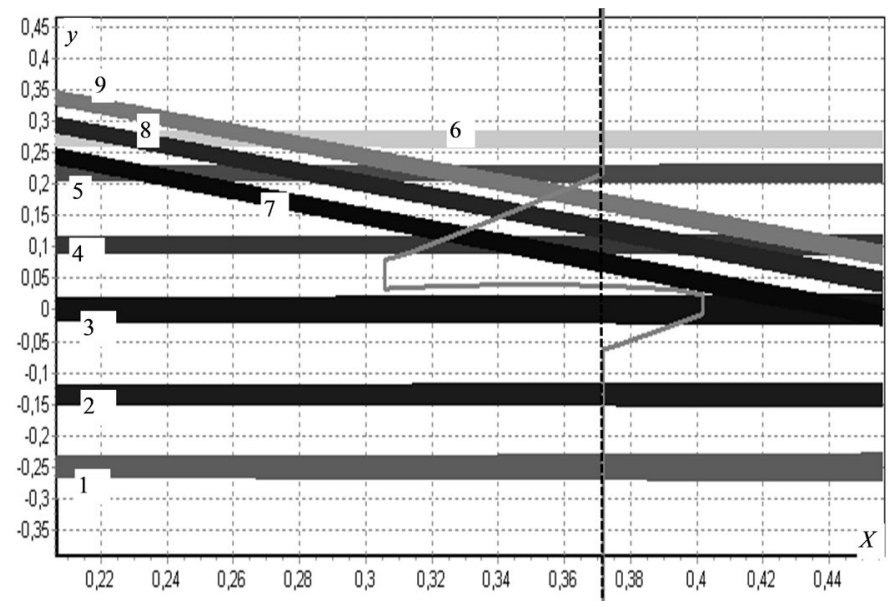

Рис. 3. Фрагмент ДКХС в области изократических режимов, не приводящих к требуемому разделению. Показана траектория градиентного режима-линзы

(сплошная линия), удовлетворяющего критерию $\mathrm{R}=1$, и наилучшего изократического режима в этой области (пунктирная линия) с неразделенными парами компонентов $(7,4)$ и $(4,8)$.

Fig. 3. Fragment of DMCS in the area of isocratic regimes that do not lead to the required separation. The trajectory of the lens gradient mode (solid line), satisfying the criterion $\mathrm{R}=1$, and the best isocratic mode in this area (dashed line) with unseparated pairs of components (7.4) and (4.8) are shown.

Алгоритм ее построения может быть следующим.

1. На ДКХС низкого разрешения - когда полосы строятся для малых значений $R_{i}^{\prime}=R_{i} / a, a>1$ (т.е. полосы получаются уже, чем требуется), находят разрешенные интервалы для изократики; 
2. выбирают крайний правый из них, удовлетворяющий условию быть не более ширины вертикальной полосы для траектории (правая граница такого интервала показана на рис.3 пунктирной линией);

3. строят ДКХС высокого разрешения - для больших $R_{i}^{\prime \prime}=a R_{i}$, и через точки пересечения полос критичных компонентов проводят траекторию;

4. рассчитывают хроматограмму и корректируют величину множителя $a$ при построении полос компонентов так, чтобы получить требуемое разрешение пиков.

Смысл алгоритма заключается в построении на запрещенных для изократики участках ДКХС траектории, огибающей узлы, в которых полосы компонентов пересекаются. Соответствующий знакопеременный градиентный режим приводит к улучшению разделения при фиксированном времени и поэтому носит название «Линза».
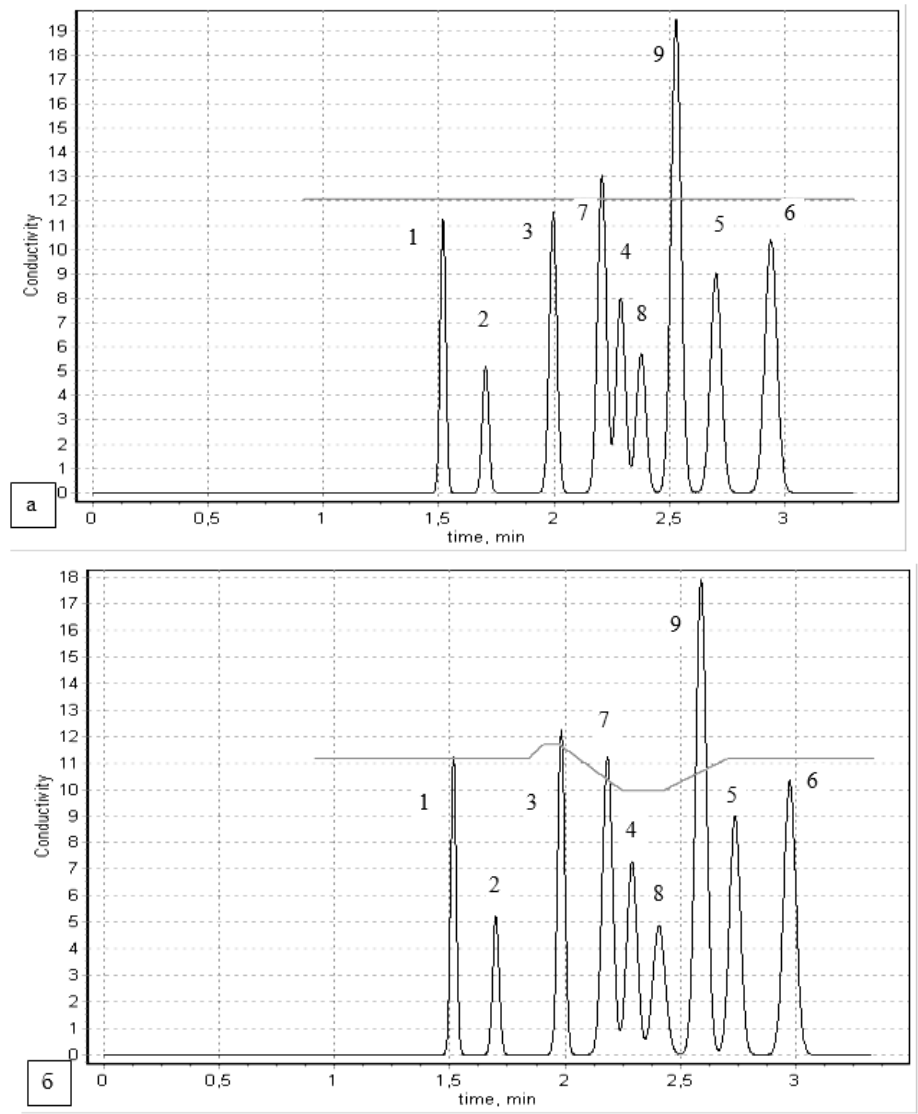

Рис. 4. (а) Хроматограмма для изократического режима, соответствующего пунктирной линии на ДКХС (рис.3). (б) Хроматограмма для слабого градиентного режима-линзы, обозначенного там же сплошной линией. Обозначения и параметры хроматографической системы указаны в подписи к рис.1.

Fig. 4. (a) Chromatogram for the isocratic regime corresponding to the dotted line on DMCS (Fig. 3). (b) Chromatogram for a weak gradient lens mode, indicated in the same place by a solid line. The designations and parameters of the chromatographic system are indicated in the caption to Fig. 1.

На рис.3 предложенный подход проиллюстрирован примером режима линзы (сплошная траектория) с низкой пиковой емкостью и удобного тем, что после анализа не требует перевода системы в состояние начального элюента (такая операция характерна для градиентных режимов и удлиняет период анализа). На рис.4 приведены расчетные хроматограммы: (а) для изократического режима, показанного пунктир- 
ной линией на рис.3, и (б) для режима линзы (там же). Во втором случае, в отличие от первого, достигается требуемое разделение пиков, причем без роста времени анализа. Сравнивая рис. 26 и 4, придем к выводу, что слабый градиентный режим «змейка» повышает производительность анализа смесей рассмотренного типа почти вдвое по сравнению с лучшим изократическим режимом.

\section{Заключение}

Итак, в работе показано, что, несмотря на чрезвычайную сложность, теоретическое описание градиентных режимов ионной хроматографии возможно с приемлемой точностью. Развитый подход к описанию сложных режимов ИХ позволяет выявить новые тонко настраиваемые условия (например, знакопеременные градиентные режимы типа «линзы»), повышающие производительность хроматографии. Достоинством предложенного режима «линза» является равенство состава элюента в начале и в конце процесса, что позволяет исключить регенерацию колонки для повторного анализа. К трудностям таких режимов можно отнести необходимость очень точного расчета и выполнения функции смешения, задающей зависимость силы элюента от времени. Решению подобных проблем служит имитационное моделирование, в результате которого создана компьютерная программа IONCHROM, позволяющая спрогнозировать поведение системы в найденных условиях.

Работа выполнена при финансовой поддержке РФФИ (грант 18-03-00382a).

\section{Список литературы}

1. Схунмакерс П. Оптимизация селективности в хроматографии / Пер. с англ. Под ред. В.А. Даванкова. М. Мир. 1989. 399 с.

2. Руденко Б.А., Руденко Г.И. Высокоэффективные хроматографические процессы: В 2 т. М. Наука. 2003. Т.1: Газовая хроматография. $425 \mathrm{c}$.

3. Kaiser R.E. // Z. Anal.Chem. 1962. Vol. 189. pp. 1-9.

4. Долгоносов А.М. // Журн. аналит. химии. 2019. Т. 74. № 4. С. 279-284. DOI: $10.1134 / \mathrm{S} 0044450219040042$

5. Долгоносов А.М., Сенявин М.М., Волощик И.Н. Ионный обмен и ионная хроматография. М. Наука. 1993. 222 с.

6. Долгоносов А.М., Ипполитова О.Д. // Журн. аналит. химии. 1993. Т. 48. № 8. С. 1361-1372.

7. Прудковский А.Г., Долгоносов А.М. // Журн. аналит. химии. 1999. Т. 54. № 2. С. 118-122.

8. Прудковский А.Г., Долгоносов А.М. Программа для моделирования ионной хроматографии IONCHROM. Патент № 2000610520 (РФ), выд. 19.06.2000.
9. Долгоносов А.М., Прудковский А.Г. // Журн. аналит. химии. 2002. Т. 57. № 12. С. 1276-1283.

10. Dionex Catalog, www.dionex.com

11. Snyder L.R., Dolan J.W., Highperformance gradient elution (The practical application of the linear-solvent-strength model). Wiley \& Sons Inc. Hoboken. New Jersey. 2007.

12. Madden J.E., Avdalovic N., Haddad P.R. et al. // J. Chromatogr. A. 2001. Vol. 910. pp. 173-179.

13. Прудковский А.Г. // Журн. аналит. химии. 2008. Т. 63. № 2. С. 184-188.

14. Долгоносов А.М., Прудковский А.Г., Колотилина Н.К. // Журн. аналит. химии. 2007. T. 62. № 11. С. 1162-1171.

15. Прудковский А.Г. // Доклады АН. 2013. T. 453. № 4. С. 373-377

16. Прудковский А.Г. // Сорбиионные $и$ хроматографические процессы. 2011. Т. 11. № 3. C. 323-334.

17. Прудковский А.Г. // Сорбичонные и хроматографические проиессы. 2012. Т. 12. № 5. C. $635-649$

18. Прудковский А.Г. // Вычислительные методы и программирование. 2013. Т. 14. 
Разд. 1. С. 390-397 (http://nummeth.srcc.msu.ru/).

19. Долгоносов А.М., Прудковский А.Г. // Доклады АН. 2013. Т. 449. № 3. С. 295-299. DOI: $10.7868 / \mathrm{S} 0869565213090132$

20. Долгоносов А.М., Рудаков О.Б., Прудковский А.Г. Колоночная аналитиче- ская хроматография: практика, теория, моделирование. СПб, Лань. 2015. 468 с.

21. Колотилина Н.К., Долгоносов А.М. // Журн. неорг. химии. 1999. Т. 44. № 5. С. 698700.

\title{
Approximation of a weak gradient in the theory of gradient ion chromatography
}

\author{
(C) 2020 Dolgonosov A.M., Prudkovskii A.G. \\ Vernadsky Institute of Geochemistry and Analytical Chemistry, Russian Academy of Sciences, \\ Moscow, Russian Federation
}

\begin{abstract}
The study presents the basic relationships between the kinetic and equilibrium characteristics of gradient ion chromatography. The relationship between these characteristics with the parameters of components on the dynamic map of the chromatographic system was found. Equations were obtained for the trajectory displaying the course of a chromatographic experiment on the map. The principles and the simplest algorithms for the procedure of optimization of the gradient ion chromatography were formulated. It was shown that, despite the extreme complexity, the theoretical description of the gradient modes of ion chromatography was possible with acceptable accuracy. The developed approach to the description of complex modes of ion chromatography allowed new finely tuned conditions to be revealed (for example, sign-alternating gradient modes of the «lens» type) that increase the productivity of chromatography. The advantage of the "lens" mode proposed in the study is the equality of the eluent composition at the beginning and at the end of the process, which eliminates the regeneration of the column for re-analysis. The difficulties of such modes include the need for a very accurate calculation and implementation of the mixing function, which sets the dependence of the strength of the eluent over time. Simulation modelling is used for the solution of such problems. A computer program IONCHROM, which allows predicting the behaviour of the system under the obtained conditions, was produced as a result of the simulation. An example of a real system was developed. For this system, the «lens» mode, which is a specially organized sign-alternating gradient mode, was predicted.
\end{abstract}

Keywords: ion chromatography theory; gradient mode; optimization of separation.

\section{References}

1. Schoenmackers P.J., Optimization of Chromatographic Selectivity. Journal of Chromatography Library, Vol. 35,. Elsevier Sci. $1986.344 \mathrm{p}$.

2. Rudenko B.A., Rudenko G.I., Vysokoeffectivnye khromatograficheskie processy, Vol. 1, Gazovaya khromatografia, M., Nauka, 2003, 425 p.

3. Kaiser R.E., Z. Anal.Chem., 1962, Vol. 189, pp. 1-9.

4. Dolgonosov A.M., J. Anal. Chem., 2019, Vol. 74, No 4, pp. 376-381. DOI: 10.1134/S106193481904004X

5. Dolgonosov A.M., Senjavin M.M., Voloshchik I.N., Ionnyi obmen i ionnaya khromatografia, M., Nauka, 1993, 222 c.
6. Dolgonosov A.M., Ippolitova O.D., $Z h$. Anal. Khim., 1993, Vol. 48, No 8, pp. 13611372.

7. Prudkovskii A.G., Dolgonosov A.M., $Z h$. Anal. Khim., 1999, Vol. 54, No 2, pp. 118-122.

8. Prudkovskii A.G., Dolgonosov A.M. Programma dlya modelirovania ionnoy khromatografii IONCHROM. Patent RF № 2000610520. 19.06.2000.

9. Dolgonosov A.M., Prudkovskii A.G., $J$. Anal. Chem., 2002, Vol. 57, No 12, pp. 10891096.

10.Dionex Catalog, www.dionex.com

11. Snyder L.R., Dolan J.W. Highperformance gradient elution (The practical application of the linear-solvent-strength model). 
Wiley \& Sons Inc. Hoboken. New Jersey. 2007. $461 \mathrm{p}$.

12.Madden J.E., Avdalovic N., Haddad P.R. et al., J. Chromatogr. A, 2001, Vol. 910, pp. 173179.

13.Prudkovskii A.G., J. Anal. Chem., 2008, Vol. 63, No 2, pp. 166-170.

14.Dolgonosov A.M., Prudkovskii A.G., Kolotilina N.K., J. Anal. Chem., 2007, Vol. 62, No 11, pp. 1046-1054.

15.Prudkovskii A.G., Doklady Math, 2013, Vol. 453, No 4, pp. 700-704.

16.Prudkovskii A.G., Sorbtsionnye i khromatograficheskie protsessy, 2011, Vol. 11, No 3, pp. 323-334.

Долгоносов Анатолий Михайлович - д.х.н., вед. научный сотрудник, лаб. сорбционных методов, ГЕОХИ РАН, Москва

Прудковский Андрей Гаральдович - д.ф.м.н., вед. научный сотрудник, лаб. сорбционных методов, ГЕОХИ РАН
17.Prudkovskii A.G., Sorbtsionnye i khromatograficheskie protsessy, 2012, Vol. 12, No 5, pp. 635-649.

18.Prudkovskii A.G., Vychislitel'nye metody $i$ programmirovanie, 2013, Vol. 14, No 1, pp. 390-397 (http://num-meth.srcc.msu.ru/).

19.Dolgonosov A.M., Prudkovskii A.G., Doklady Chem., 2013, Vol. 449, Part 1, pp. 8993. DOI: $10.7868 / \mathrm{S} 0869565213090132$

20.Dolgonosov A.M., Rudakov O.B., Prudkovskii A.G. Kolonochnaya analiticheskaya khromatografia: praktika, teoriya, modelirovanie. SPb. Lan', 2015, 468 p.

21.Kolotilina N.K., Dolgonosov A.M., Rus. $J$. Inorg. Chem., 1999, Vol. 44, No 5, pp. 803-805.

Dolgonosov Anatoly M. - Dr. Sci. (Chem.), Lead. research sci., Lab. of sorption methods, GEOKHI RAS, Moscow6 amdolgo@mail.ru

Prudkovskiy Andrey G. - Dr. Sci. (Phys.-Math.) Lead. research sci., Lab. of sorption methods, GEOKHI RAS, Moscow 